\title{
On the time-invariance properties of upper limb synergies
}

\author{
Giuseppe Averta*1,2,3, Student Member, IEEE, Gaetano Valenza ${ }^{1,3}$, Senior Member, IEEE, \\ Vincenzo Catrambone ${ }^{1,3}$, Student Member, IEEE, Federica Barontini ${ }^{2}$, \\ Enzo Pasquale Scilingo ${ }^{1,3}$, Senior Member, IEEE, Antonio Bicchi ${ }^{1,2,3}$, Fellow, IEEE, \\ Matteo Bianchi ${ }^{1,3}$, Member, IEEE,
}

\begin{abstract}
In this work, we present a novel approach to dynamically describe human upper limb trajectories, addressing the question on whether and to which extent synergistic multijoint behavior is observed and preserved over time evolution and across subjects. To this goal, we performed experiments to collect human upper limb joint angle trajectories and organized them in a dataset of daily living tasks. We then characterized the upper limb poses at each time frame through a technique that we named Repeated-Principal Component Analysis (R-PCA). We found that, although there is no strong evidence on the predominance of one Principal Component (PC) over the others, the subspace identified by the first three PCs takes into account most of motion variability. We evaluated the stability of these results over time, showing that during the reaching phase there is a strong consistency of these findings across participants. In other words, our results suggest that there is a time-invariant low dimensional approximation of upper limb kinematics, which can be used to define a suitable reduced dimensionality control space for upper limb robotic devices in motion phases.
\end{abstract}

\section{INTRODUCTION}

$\mathbf{T}$ HE human nervous system can manage the extraordinary kinematic complexity of the muscular-skeletal apparatus, enabling the seamless and effective execution of different motor actions. Understanding the processes underpinning this behavior represents a very active and fascinating topic in neuroscience, where a lot of attention has been devoted to the investigation of the concept of synergies, with a special focus on the hand (see next section for a discussion on the related work). Synergies can be regarded as principal control patterns that the brain rely on to cope with the abundancy of the degrees of freedom (DoFs), for motion generation [1]. The study of this generalized simplification approach has shed light on the neural mechanisms of kinesiology and also opened promising perspectives e.g. in assistive/rehabilitation robotics and prosthetics [2]-[6], for the design and control of human-inspired artificial devices with a reduced number of inputs [7].

Looking at the human upper limb, the investigation of the underpinning synergy-based control and its characteristics

This project has received funding from the European Union's Horizon 2020 research and innovation programme under grant agreement No. 688857 (SoftPro).

*Corresponding Author: giuseppe.averta@ing.unipi.it

1 are with Centro di Ricerca "Enrico Piaggio", Universita" di Pisa, Largo Lucio Lazzarino 1, 56126 Pisa, Italy giuseppe.averta@ing.unipi.it

${ }^{2}$ are with Fondazione Istituto Italiano di Tecnologia, via Morego, 30, 16163 Genova, Italy

3 are with Dipartimento di Ingegneria dell'Informazione, Universita ‘ di Pisa, via G. Caruso no 16,56122 Pisa, Italy over time has received little attention so far, compared with analogous studies on human hand postural synergies. The latter usually employ dimensionality reduction to identify principal joint covariation patterns, e.g. through Principal Component Analysis (PCA) (see next section). However, PCA cannot be applied for characterizing time-varying phenomena, since the assumption of Independent and Identically Distributed (i.i.d.) samples would be violated [8]. State-of-the art methods to enable the use of PCA with temporal kinematic data usually rely on the augmentation of the dimensionality of the dataset, expanding the number of samples along the temporal coordinate [910]. However, in this way, the computed principal components take into account trajectory variability, rather than inter-joint coupling. Furthermore, when the temporal steps considered in the analysis become numerous, the dimensionality of the problem also increases, and the number of experiments required to guarantee that the PCA is well posed makes this implementation practically unfeasible or, at least, highly challenging.

To overcome these limitations and bridge the gap between hand and upper limb synergy investigation, in this work, we propose a new approach, named Repeated PCA (R-PCA), which applies PCA on upper limb joint angles, at each time frame of the motion trajectories. More specifically, after a preprocessing phase based on Dynamic Time Warping, principal components are calculated for each temporal frame of motion execution. In this manner, we do not violate the underpinning applicability hypotheses of PCA, since the samples from different temporal steps can be regarded as i.i.d. To this goal, we built a dataset of motor tasks, by asking 33 right-handed able bodied participants (17 Female, age $26.56 \pm 2.77$ ) to perform 30 daily living actions with their arm, belonging to three different classes [11]. We evaluated the in-time stability of these components by quantifying the angular deviation between the subspaces identified by a reduced number of PCs at each frame. Surprisingly, despite the non-negligible variability of the single component in time, the norm of the 3D angles between the subspaces identified by the first three PCs remains lower than 20 degrees for all the motion phases, increasing only during the contact-manipulation one. These findings suggest that human upper limb movements can be generated through a time-invariant model with a good approximation during motion phases. Such an approximation degrades when the hand is in contact with the environment or is adapting to perform a specific task. To verify these results, we report a two-stage 
validation: (i) a cross-validation, where we repeated 10-times our analysis on a random subset of 20 subjects; (ii) validation on a synthetic dataset that we obtained by fitting a time invariant statistical model on real data. Results show that our description appears robust across subjects and coherent with the analytic outcomes obtained with the synthetic data. The latter point allows to exclude possible effects due to the pre-processing algorithms in use. Moreover, the results on the synthetic dataset further characterize the proposed measure as a quantification of the time-invariance properties of the analyzed phenomenon during the motion phases.

\section{RELATED WORK}

The investigation of the synergistic organization in the motor domain has been tackled from different points of view and at various levels (including muscular and kinematic), with a special focus on the hand [7]. The main outcomes of all these studies is that the number of DoFs directly controlled by the human nervous system is lower than the one resulting from a pure mechanical count [12]. Latash and colleagues [13] defined synergies as a neural organization resulting in task specific covariation of elemental variables, with the ultimate goal of generating stable and effective movements. This theory, also known as Uncontrolled Manifold [13 14], suggests that unit elements are not individually controlled but rather are free to vary. This fact is hence exploited by the nervous system to minimize the error in endpoint control and maximize motion performances. The three main mechanisms identified in [13] for implementing the synergistic control are: i) the elimination of specific kinematic DoFs (e.g. using muscles spanning several kinematic joints [15]); ii) the optimization of specific cost functions (e.g. end effector jerk) to get coordinated movements [16 17]; iii) the functional linkage between unit elements ([18]-[23]. The implication of the latter point at the level of muscle activation led to the introduction of the concept of m-modes, i.e. groups of muscles controlled together [24], which act as single functional units. At the muscular level, the concept of synergy-based dimensionality reduction has received considerable attention and produced numerous relevant results, leading to the identification of muscle synergies as the building blocks for natural motion generation [20]. The general approach is to record ElectroMyoGraphic (EMG) signals from a large set of muscles during the execution of specific tasks. Matrix factorization techniques are then used to identify patterns of muscles co-activations, associated to independent synergies, which are then related to relevant action variables [25]. In [20], the authors affirmed that these components are actively enrolled, following task-specific strategies, although some muscle activation patterns are preserved across tasks. Notwithstanding the very reduced number of synergies observed in these tasks, a high variability of the muscle activity profile can be generated by combining muscle synergies, modulating their amplitude and temporal structure [26]. The existence of muscle synergies in humans was confirmed by several other studies, e.g. during static hand postures [27 28], for active force generation with one or more fingers [29]-[31], and at the upper limb level during fast reaching movements [32].
Similar outcomes were observed at the lower limb level, e.g. in [33] the authors demonstrated that the EMG signals from 32 muscles in humans during walking are organized in patterns of temporal activation of five independent components. Alterations in this synergistic description were observed in case of motion impairment, e.g. as a consequence of stroke events. In [34], analyzing chronic stroke survivors performing a task of 3-D force matching, the authors observed the recruitment of altered shoulder muscle synergies, which was strongly associated to abnormal task performance. In [35] the authors proposed a index of impairment level based on the difference, between the affected and non-affected upper limb, in terms of movement complexity, calculated as the cumulative of variance explained by functional synergies. In [36] the authors reported on trainingrelated changes in synergies while using a robotic therapy device to train and analyze impaired arm movements. For an overview on these topics the interested reader may refer to 37.

At the kinematic level, the synergistic description was mainly studied looking at the hand. Besides the well known papers of Santello and colleagues on hand postural synergies during grasp of imagined and real objects [18 19], several other studies on this topic can be found in literature (see e.g. to [938][41]). In these papers, dimensionality reduction techniques, namely PCA, Non Negative Matrix Factorization, etc., were applied to datasets of hand poses (joint values) recorded during specific tasks. Results show that a reduced number of main correlation patterns between hand joints, e.g. described in terms of PCs, explains most of pose variability (these results were then used to develop under-actuated robotic hands that implemented these principal co-variation schemes, e.g. directly embedded in the mechanical design see e.g. [42]). However, to the best of authors' knowledge, the rest of upper limb has not received the same level of attention, despite the importance that it plays to effectively orientate the hand for a successful task accomplishment. The attention was mainly devoted to the comparative analysis of pathological and physiological conditions, as in [43], where upper limb movements were studied during selected tasks to compare stroke patients and able-bodied participants. PCA was used on one healthy subject and one stroke patient performing different movements: cross grasping, power grasping and pointing gestures. Authors reported on some similarities and differences between stroke and non-stroke synergies. Similarly, in [44] it was observed that persons with hemiparesis exploit the redundancy of their motor control to stabilize performance variables, in a way similar to healthy subjects - although with different patterns of joint couplings. In [45], a quantitative method to assess upper limb motor deficits in children with cerebral palsy using three-dimensional motion analysis during the reach and grasp cycle was developed. Other papers studied muscular synergistic activation in upper limb, as in [46], where electromyographic activity was recorded from shoulder and arm muscles during point-to-point movements. However, the relation between the synergistic description at muscle level and its impact on the kinematics is still under investigation [47]. 
A

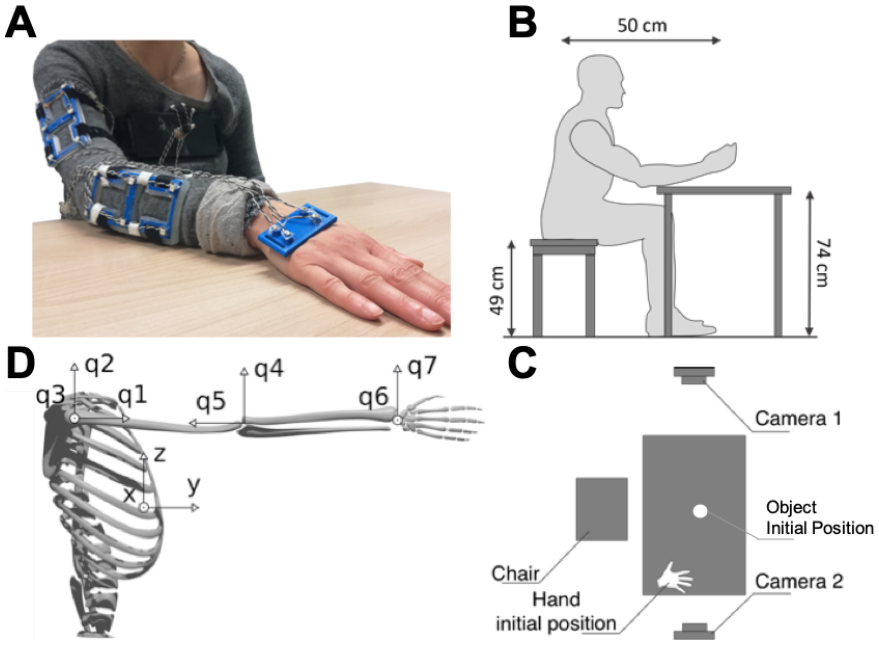

Fig. 1. In figure A we show marker location on upper limb and chest, in figure $\mathrm{B}$ and $\mathrm{C}$ we report a scheme of the experimental setup. Two RGB cameras are included to record the scene. Finally, in figure D, we show the system parametrization. The labels $q_{1}, \ldots, q_{7}$ refer to the joint angles of the model. The global reference frame is fixed to the chest (epigastrium) and defined by the axes $x, y$ and $z$.

\section{EXPERIMENTAL SETUP}

\section{A. Setup and Experiments}

A comprehensive study of human upper limb control requires a large dataset of movements, i.e. a set of actions that span the whole upper limb workspace performed by different subjects [18 39948$]$. In this work, we asked 33 subjects (17 Female, age $26.56 \pm 2.77$, all right-handed) to perform a set of 30 different daily-living tasks and recorded the 3D position of 20 active optical markers fastened to the upper limb and the chest using a Phase Space system (sampling frequency $100 \mathrm{~Hz}$ ) - see Fig. 1. A redundant marker set was used to improve tracking performance and avoid occlusion problems. In particular, we placed 4 markers fixed to the chest, 6 markers fixed to the lateral arm, 6 markers fixed to the dorsal forearm and 4 markers fixed to the hand dorsum. Each marker set was placed on an ABS-printed support, whose position was estimated during the participant-specific calibration procedure, described in the following.

The choice of the experimental tasks was inspired by state of the art grasping taxonomies [4950], and the analysis of human upper limb movement workspace [51]-[53]. It is worth mentioning that, although the hand kinematics is not considered in the actual analysis, the final grasping pose determines the control of the rest of the upper limb [39]. In addition to the aforementioned purely kinematic considerations, we selected the experimental tasks according to three main classes that are known to produce different activation patterns at the brain cortex level [54]. The twofold motivation for this choice is related to both the need to differentiate arm movements based on task purpose [18], and to comparatively analyze the kinematic outputs with the brain activity, which we also recorded using an electroencephalogram (EEG) system [11]. The long term objective is to devise design guidelines for the development of robust brain machine interfaces - the latter point is out of the scope of this work and hence it will not be further considered. The three classes consist of 10 actions each, and can be defined as: i) intransitive, i.e. actions that do not need the usage of any object; ii) transitive, i.e. actions that require the use of an object; iii) tool-mediated, i.e. actions that use an object to interact with another one. Tasks were meant to be executed in a random order, three times each, with participants' dominant hand. The subject seated on a chair, with the hand in a defined starting position and the objects placed on a frontal table at fixed distances (see Fig. 1). At the end of each task, the subject returned to the starting point. Subject were naive about the purpose of the study, and had no history of neuromuscular or cognitive disorders that would have affected the experimental outcomes. Each participant signed an informed consent to participate in the experiment, while the experimental protocol was approved by the Institutional Review Board of the University of Pisa, in accordance with the declaration of Helsinki. The complete list of movements is reported in Appendix A.

\section{B. Movement Identification}

a) Modeling of upper limb kinematics: The description of human kinematics is in general very complex. Several models have been proposed in literature (the interested reader can refer to [55 56], just to cite a few). In this work, we took inspiration from [55], adopting a 7 DoFs and 3 rigid link timeinvariant model. We already used this description in [57], and it was proven to represent a good trade-off between modeling complexity and accuracy. Joint angles are named as $q_{1}, \ldots, q_{7}$ : $q_{1}$ is associated to the shoulder abduction-adduction; $q_{2}$ is associated to the shoulder flexion-extension; $q_{3}$ is associated to the shoulder external-internal rotation; $q_{4}$ is associated to the elbow flexion-extension; $q_{5}$ is associated to the elbow pronationsupination; $q_{6}$ is associated to the wrist abduction-adduction; $q_{7}$ is associated to the wrist flexion-extension. In Fig. $11 \mathrm{D}$, we report a schematics of the kinematic model.

The kinematics is hence completely defined through a set of parameters, which are evaluated for each subject using a calibration procedure. In the following subsections we briefly summarize the techniques used for model calibration and joint angle estimation. For further details on the kinematic model adopted in this work and the procedures for motion estimation, the interested reader is invited to refer to [57].

b) Model calibration and joint angle estimation: Considering the high variability of kinematic parameters among subjects, we designed a first procedure to calibrate the model for each specific participant, by calculating an optimal parameter set. This is achieved by solving the following constrained least-squares minimization problem:

$$
\left(x^{*}, p_{G}^{*}\right)=\arg \min _{x_{k} \in D_{x}, p_{G} \in D_{p}} \frac{1}{2} \sum_{k=1}^{N_{p}} r_{k}^{T} r_{k}
$$

where $r_{k}$ - the residual function that we aim at minimizing is calculated as the error between the measured marker position $y_{k}$ and the marker position estimated through the forward kinematics $(\mathrm{FK}) f\left(x_{k}, p_{G}\right)$; FK inputs are $x_{k}$, which represents 
the vector of the estimated joint angles, and $p_{G}$, i.e. the vector of model kinematic parameters. We assumed the following problem constraints: i) joint values are only within the upper limb range of motion and ii) parameters can vary around a preliminary estimation of parameters performed with manual measurements (initial conditions). Calibration performances are further improved by evaluating this procedure upon different time frames. This was performed by concatenating the measures of marker positions and estimations in the vectors $y_{k}$ and $f\left(x_{k}, p_{G}\right)$.

The calibrated model was then used as input for an identification procedure used to estimate the joint angles over time. This was achieved using an Extended Kalman Filter (EKF) based algorithm.

In particular, let the movement be considered as an uncertain noisy process where at time frame $k$ the joint angle vector $x_{k}$ is the state of the process, $y_{k}$ is the markers position vector, $w_{k}$ and $v_{k}$ are process and observation zero mean gaussian noises, with covariance $Q_{k}$ and $R_{k}$, respectively, and $f\left(x_{k}\right)$ is the forward kinematics. Note that, once the calibration phase is completed, the vector $p_{G}$ is a known parameter of the model, hence the FK depends only on the values of joint angles $x_{k}$.

The system can be described using the following equations:

$$
\left\{\begin{array}{l}
x_{k}=x_{k-1}+w_{k} \\
y_{k}=f\left(x_{k}\right)+v_{k}
\end{array}\right.
$$

Given the state at time frame $k-1$, the state at time $k$ is calculated through a 2 -step procedure:

- prediction of the future state $\hat{x}_{k \mid k-1}=\hat{x}_{k-1}$;

- update of the state estimated in the first step by calculating $\hat{x}_{k \mid k}=\hat{x}_{k \mid k-1}+K_{k} \tilde{r}_{k}$.

The amount of correction of step 2 is calculated as the product between the error vector $\tilde{r}_{k}=y_{k}-f\left(\hat{x}_{k \mid k-1}\right)$ and the matrix $K_{k}$, where $K_{k}=P_{k \mid k-1} H_{k}^{T} S_{k}^{-1}$ is the Kalman Gain, $P_{k \mid k-1}$ is the covariance matrix estimation of the predicted state, $H_{k}=$ $\frac{\partial(f(x))}{\partial(x)}$ is the Jacobian matrix and $S_{k}=H_{k} P_{k \mid k-1} H_{k}^{T}+R_{k}$ is the residual covariance.

\section{DATA ANALYSis}

To answer the question on whether and to which extent a lowdimensional representation of upper limb trajectories and interjoint couplings exists, one way could be to use a dimensionality reduction technique, such as Principal Component Analysis (PCA). PCA has been extensively used in the state of the art of motion analysis (see section Related Work) to identify principal directions of data variance, which may be intended as the principal DoF coupling scheme, which explain most of pose variability (see e.g. [18 19 |38]). As reported in the Introduction and Related Work sections, several studies have focused on hand investigation, while the rest of upper limb has often been discarded. In addition, time-dependence of joint trajectories is scarcely considered when using standard PCA [10]. Indeed, the latter point must deal with the underlying observation that kinematic description at time step $i$ intrinsically depends on the previous poses of the upper limb. As discussed in [8], classic PCA assumes that the elements of the datasets are i.i.d. This essentially excludes the usage of temporally-correlated samples.
To overcome this limitation, yet preserving the temporal information, we introduce a new methodological application of PCA that we have named Repeated-PCA (R-PCA). RPCA allows to compute time-varying PCs while a descriptive approach - reported later - enables to evaluate the robustness of PC-based kinematic description over time, through a frame-byframe result comparison. To correctly implement this approach, it is mandatory to have kinematic recordings synchronized in time. In other terms, taken one sample as reference, all the acquisitions must be modified in such a way to match the same start and stop frame. This is typically solved by applying Dynamic Time Warping (DTW)[57] to the dataset consisting of upper limb joint evolutions recorded during the experimental tasks of the protocol. DTW is a technique that reshape temporal evolutions so as they are synchronized, i.e. they have the same duration and timing. Before DTW, a moving average filter (window width 10 samples) was applied to each element of the dataset.

\section{A. Dynamic Time Warping}

Temporal synchronization between signals is typically used to increase sample affinity by conforming starting-time and velocity of action execution [58 59]. Under a mathematical point of view, this can be regarded as the problem of finding the optimal time-shift and time-stretch between signals, or, in other terms, a dynamic warping in time to maximize inter-signal correlation. In this work, we implemented DTW as in [57], leveraging on the following assumptions: time-monotonicity, to preserve the coherence in time of the sample; linear distortion in time, i.e. including a scaling factor to the time domain and, hence, to the action velocity.

Given two time series, $v_{1}$ and $v_{2}$, the affinity between the two signals is maximized by solving the following optimization problem:

$$
(S, T)=\underset{S>0, T}{\operatorname{argmax}} \rho\left(v_{1}(t), v_{2}(S t-T)\right)
$$

where the operator $\rho$ is the cross correlation between two vectors. Given two generic vectors $A, B \in \mathbb{R}^{N}, \rho$ is calculated as:

$$
\rho(A, B)=\frac{1}{N-1} \sum_{i=1}^{N}\left(\frac{A_{i}-\mu_{A}}{\sigma_{A}}\right)\left(\frac{B_{i}-\mu_{B}}{\sigma_{B}}\right)
$$

where $\mu_{A}$ and $\sigma_{A}$ are the mean and standard deviation of $A$, and $\mu_{B}$ and $\sigma_{B}$ are the mean and standard deviation of $B$, respectively. Problem 2 optimizes two parameters: $S$, i.e. the scaling factor applied to the velocity of signal $v_{2}$; and $T$, i.e. the time shifting applied to $v_{2}$.

DTW was applied to the whole dataset of joint trajectories, having as reference signal (with respect to which the optimization is carried out) one random elements of the dataset. In this way we can obtain the parameters $S$ and $T$ corresponding to all the recorded samples. The set of parameters identified with such procedure was then used to warp all the joint signals coherently. After this step, all the elements have the same number of elements (i.e. same time-frames) and are represented 
in a matrix $\in R^{N_{t} m}$, where $N_{t}$ is the number of time frames considered and $m$ is the number of DoFs.

\section{B. Repeated Principal Component Analysis}

As previously stated, PCA represents one of the most common approaches to identify principal patterns of variation within a dataset. PCA computes the orthogonal transformation that converts a set of correlated observations into a set of linearly uncorrelated variables called Principal Components (PCs). In other terms, let us consider $X$ to be the matrix $\in R^{N \times m}$ that collects $N$ observations from a stochastic process with $m$ degrees of freedom. Principal Components (PCs) can be calculated as the eigenvectors $S_{i}$ of the covariance matrix of data $\Sigma=X X^{T}-\mu \mu^{T}$, where $\mu=\operatorname{mean}(X)$. When applied to the description of kinematic postures, these vectors are typically named Postural Synergies [18]. The amount of data variability that each PC can explain is calculated as the normalized corresponding eigenvalue. In other terms, the first synergy is the vector $S_{1}$ that maximizes the function $\sum_{i} f_{i 1}^{2}$ subject to $\left\|S_{1}\right\|=1$, where $f_{i 1}=S_{1}^{T} S_{i}$ is the first principal component score; the second eigenvector $S_{2}$, then, maximizes $\sum_{i} f_{i 2}^{2}$ subject to $\left\|S_{2}\right\|=1$ and $S_{2}^{T} S_{1}=0$, and so on. Note that this problem is well posed only if $N>>m$.

It is worth noticing that all the elements of the dataset are intended as single independent observations of the same stochastic process. The $i^{t h}$ row of matrix $X$ is a vector of $m$ variables. This severely limits the application of PCA for the investigation of human movements, given the intrinsic timecorrelation of the motion samples (see Introduction). For this reason, a lot of studies on PCA for motion analysis focus on single frames of the movement, typically the last one, i.e. the final grasp pose (see eg. [18|19|39]), discarding the temporal evolution of the signals.

In this work, we propose an innovative approach to include the temporal information into PCA, while preserving the dimensionality of the problem. This algorithm, which is described in the following, is named Repeated Principal Component Analysis (R-PCA).

Let us consider $X_{i}$ to be the matrix $\in \mathbb{R}^{N \times m}$ that collects the upper limb poses of all the $N$ elements of the dataset for the specific time frame $i$. In this case, $X_{i}$ collects a set of i.i.d. samples in $R^{m}$ and, then, it can be characterized using the classic PCA implementation previously described in this section. The outcomes of this analysis describe the principal joint couplings that emerge at the specific time-frame $i$. Consequently, repeating this analysis while varying $i$ between 1 and $N_{t}$ allows to describe the temporal evolution of the synergistic behavior.

It is, in general, plausible that the synergistic coupling between joints is not constant in time. To evaluate the variation of PCs during a task execution, it is possible to quantify the difference between the subspaces spanned by time-varying PCs and the subspaces spanned by a corresponding set of synergies used as reference. Without any loss of generality, here we used as reference synergies the PCs calculated considering the average poses (over time) during motion, over the whole dataset. Hereinafter, we will refer to these PCs as global static synergies. Note that the mean pose is used only as a singleframe representation of the movement. In particular, in case of 2 one-dimensional manifolds, defined by the vectors $v_{1}$ and $v_{2}$, the minimum angular distance between them is quantified as $\theta_{1,2}=\cos ^{-1}\left(v_{1}^{T} v_{2}\right)$. This is the typical case in which we want to compare two single synergies (i.e. vectors). In case of two or more dimensions, the distance between subspaces is provided through a number of angles equal to the cardinality of the vector set considered. In this work we rely on the definition of principal angles between subspaces given in [60]. Given the vector of the principal angles, the distance between two subspaces is finally calculated as norm of this vector.

\section{RESULTS}

In Fig. 2 we report the variance explained in time by the first three PCs. While for hand movements several studies identified one principal component that explains a considerable part of dataset variability, our results on upper limb seem suggesting that there is no strong dominance of one inter-joint coupling scheme among the others, but rather we can identify three main PCs that, together, explain between $70 \%$ and $85 \%$ of the total variance $(73.13 \pm 4.64 \%)$. The variance explained by each PC is almost stable over time, respectively $36.07 \pm 3.24 \%$, $24.37 \pm 1.79 \%, 16.68 \pm 2.44 \%$. These values indicate that the Euclidean space defined by the first three components represents a low dimensional control manifold which accounts for most of the upper limb movement variability.

What is also noticeable - as expected - is that the variance explained by the first three components is lower during the interaction phase, i.e. when subjects are in contact with the objects or are performing specific gestures. This observation support the idea that the complexity of motion control increases when subjects are interacting with the environment. Future works will further investigate this part, including a multisensory analysis of the hand control that will comparatively evaluate hand kinematics and EEG activation.

Looking at individual PCs computed through R-PCA, we observe that they are not stable in time, but rather present a strong variation during motion execution (see Fig. 3) which could be interpreted as a dynamic modulation along trajectories.

To systematically evaluate synergies variability in time, we used the definition of distance between subspaces introduced in IV-B. In particular, the stability of each single synergy is quantified as the time-varying distance with respect to the corresponding PC defined on the average dataset (global static synergy). The denomination "corresponding" is intended hereinafter in terms of cardinality, i.e. the first time-varying synergy is compared with the first global static synergy and so on. This definition can be easily extended to multi-dimensional manifolds: the distance between two k-dimensional subspaces is calculated as the norm of $\mathrm{k}$ principal angles, as defined in [60].

In Fig. 4 we report the angular distance between each time-varying synergy (only the first three are reported here for the sake of space), and the corresponding global static synergy. As expected, synergies show a remarkable variation in 


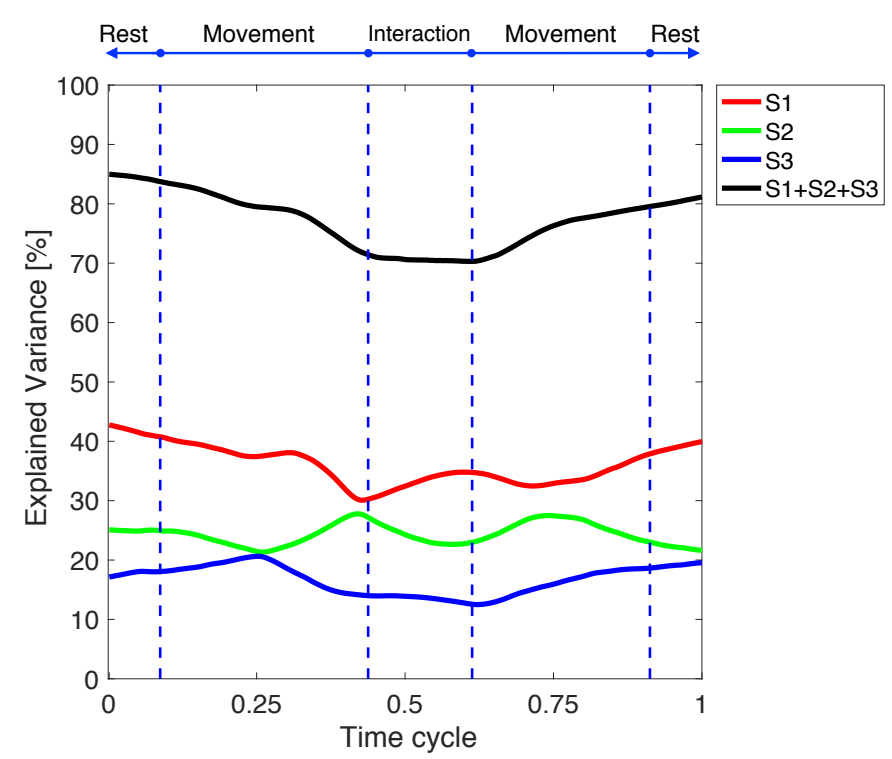

Fig. 2. Percentage of variance explained by the first three Principal Components in time. Red plot is associated to $S_{1}$, green plot to $S_{2}$ and blue plot to $S_{3}$. The sum is finally reported in black.

time - not directly correlated with the action phases. However, interestingly, when we consider the subspace defined by the first three PCs together, the norm of the 3D distance w.r.t. the corresponding global static synergy remains below 20 degrees for the whole movement phases (see Fig 5), while increases during the contact/manipulation/fine gesture control phase. This aspect seems to be coherent with the observation we have before reported regarding the variance that components explain in time. In other terms, results show that, even though single PCs present a high variability in time, the subspace of the first three PCs remains rather invariant during the pure motion phases. It is worth noticing that such effect is not present when we compare sub-manifolds with higher or lower dimensionality. We believe that, for two-dimensional manifolds (i.e. $S_{1}+S_{2}$ ), this is due to the fact that two PCS are not sufficient to describe the dataset variability in a sufficiently accurate manner and, then, the third one is needed to effectively take into account upper limb variability and control. Regarding higher-dimensional manifolds (number of components between 4 and 6), this observation remains an open point. One possible explanation could be that, while the first three synergies are used to shape the gross movement, higher order synergies account for very reduced contribution mainly used to refine the motion and, hence, can show different pattern during the temporal evolution.

\section{A. Robustness across Subjects and Validation}

We investigated the robustness of our results across subjects by repeating the aforementioned analyses 10 times, while randomly selecting a subset of 20 subjects for further analyses. Results show that the norm of three-dimensional distance between subspaces presents a shape similar to the one reported in Fig. 5 (related plots are omitted here for the sake of space), and it is always below 25 deg during the motion phases.

Moreover, to verify that the time-varying angular deviation between individual PCs (see Fig 4 ) is actually due to the
TABLE I

COEFFICIENTS OF THE FIRST THREE GLOBAL STATIC SYNERGIES.

\begin{tabular}{cccc}
\hline & $\mathbf{S}_{1}$ & $\mathbf{S}_{2}$ & $\mathbf{S}_{3}$ \\
\hline $\mathbf{q}_{1}$ & -0.5280 & 0.4504 & 0.2619 \\
$\mathbf{q}_{2}$ & 0.2898 & -0.1376 & 0.0219 \\
$\mathbf{q}_{3}$ & -0.1011 & 0.1454 & 0.0705 \\
$\mathbf{q}_{4}$ & -0.2067 & 0.2296 & -0.4727 \\
$\mathbf{q}_{5}$ & 0.3999 & -0.3577 & -0.0880 \\
$\mathbf{q}_{6}$ & 0.0967 & -0.0520 & 0.8335 \\
$\mathbf{q}_{7}$ & 0.6442 & 0.7574 & -0.0063 \\
\hline
\end{tabular}

non-stationarity of the upper-limb motor control, with no biases from the proposed R-PCA methodology, we performed the analyses on a synthetic dataset generated through an autoregressive (AR) model identified on real data. In particular, the following AR model:

$$
y(t)=a_{1} y(t-1)+\cdots+a_{n_{a}} y\left(t-n_{a}\right)+e(t),
$$

with $e(t)$ i.i.d. Gaussian white noise with zero mean and standard deviation equal to 1 , was fitted on real data (selecting one random trial from the whole dataset) at different model order. We chose $n_{a}=4$ in accordance with the Akaike information criterion and the minimal root mean square error. Parameters $a_{i}$ are then estimated through a least-square method. Then, we generated 1000 repetitions of a $7 \times 500$ realization of the AR process, and applied our R-PCA method. As the model is time-invariant, it ensures the stationarity of the output. As expected, results show negligible values of the norm of angular deviation between the first three PC-based subspace over time as compared to the global static one. Indeed, the norm of the angular deviation resulted as low as $1.3684 \pm 0.4760$ (Max $=2.338$ ).

\section{B. Principal Component description}

Results presented so far demonstrates that a static threedimensional description of upper limb kinematics is sufficient to explain a high percentage of dataset variance during different motion phases. Moreover, it is possible to affirm that such approximation can be considered effective during the free movements of the upper limb. Considering the movement phases in which the process can be intended as time-invariant, we can define a set of three vectors that are contained in the span of the first three global static synergies and use it to describe the upper limb movements through a time-invariant basis. In Fig 6 we report a visual reconstruction of an upper limb moving around the mean pose along the first three global static synergies. The three main movements are not in general required to be physically meaningful. However, it is possible to observe that the first global static synergy appears to be an extension/flexion of the arm in the sagittal plane, while the second is more involved in modulating the level of the hand along the vertical axis. Numerical values of the first three global static synergies are listed in table [1. 

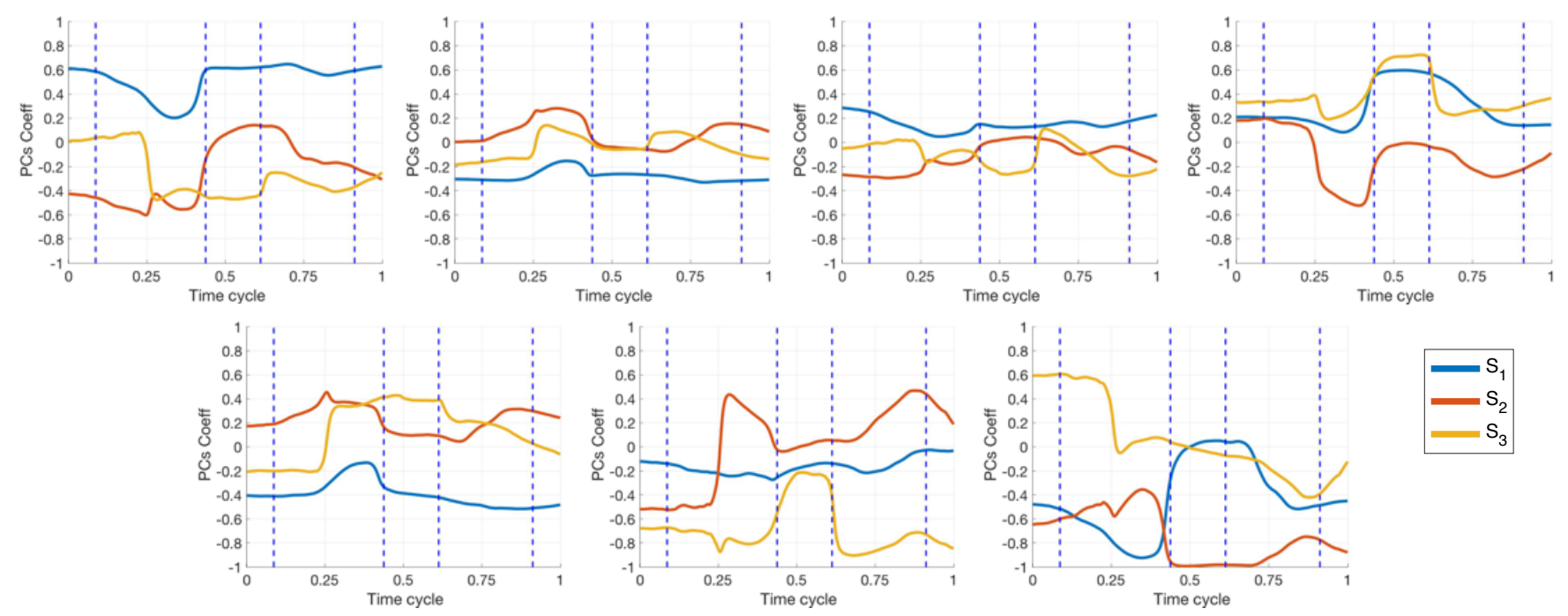

Fig. 3. Coefficients of the first three PCs in Time. From top-left to bottom-right we report the values for joints $q_{1}-q_{7}$ according to the labeling of Fig. 1 D. For reader's convenience, time cycle is segmented according to Fig. 2
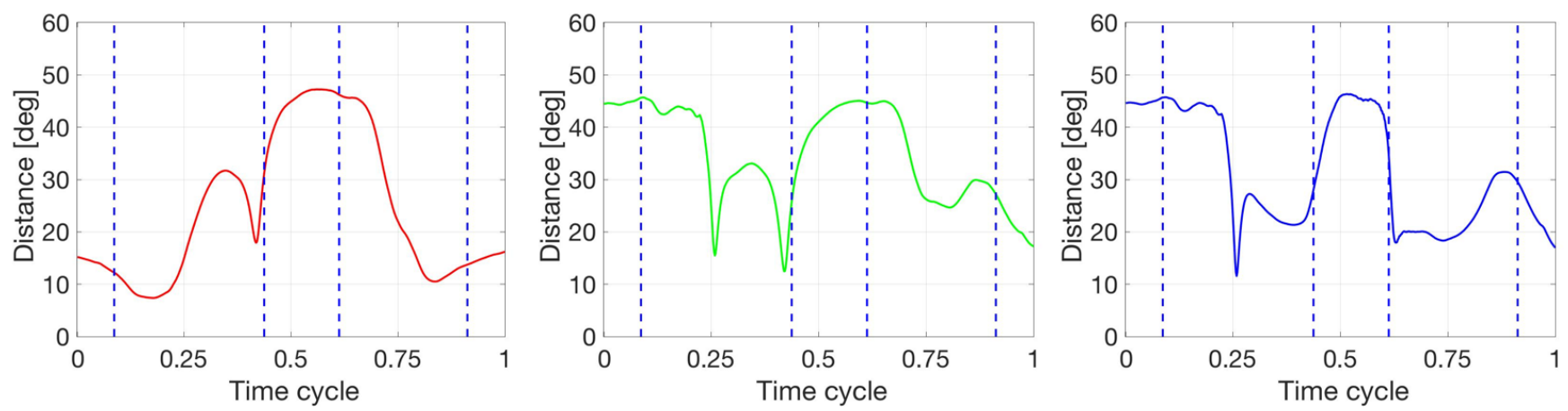

Fig. 4. Angular deviation between individual PCs computed through R-PCA and the corresponding global static PCs. From left to right, we report the values in degree - over the time for the first, the second and the third one, respectively. For reader's convenience, time cycle is segmented according to Fig. 2

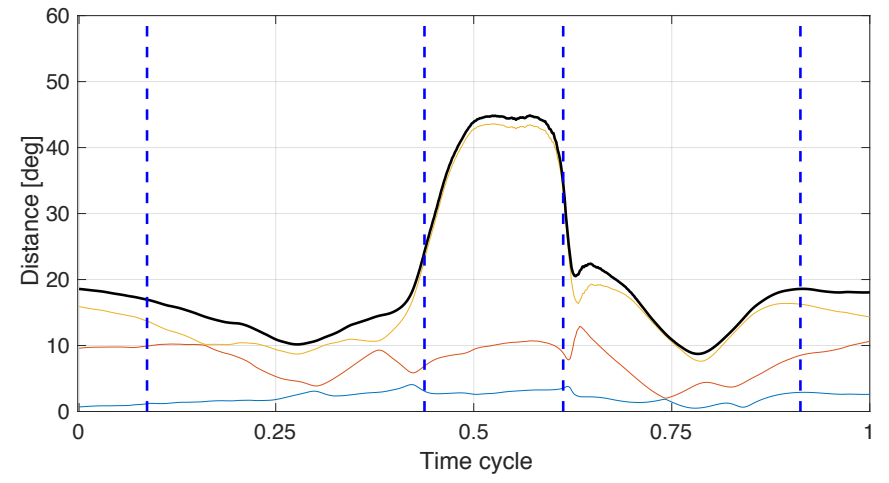

Fig. 5. Norm of 3D angular deviation between the subspace of the first three PCs computed through R-PCA and the subspace of the first three global static synergies. Yellow, red and blue lines show the three principal angles between the two subspaces as defined in [60], while in black we report their norm.

\section{Discussions}

The results presented in this study suggest that a synergistic coupling between upper limb joints can be observed during daily living activities. These synergies define a three dimensional sub-manifold that appears to be stable during the motion phase. We do believe that these outcomes can shade light on the neural mechanisms underpinning synergistic motion generation over time and potentially impact the rehabilitation field too. Indeed, neurological disorders, e.g. stroke and focal dystonia, affect the ability to coordinate multi-joint movements [61]. Having a robust and simplified description of upperlimb kinematics in physiological conditions could serve as a baseline to evaluate eventual discrepancies induced by the pathology, which can affect the PC-based low-dimensional representation of motions (different number and/or type of PCs involved), its time-invariance, and the robustness across subjects. For the latter point, it is indeed known that there is a large phenomenological variety in subjects with motor disorders (related to different levels of impairment) [61], which can be compared with the physiological case to assess the effects of rehabilitative procedures.

However, the implications of this work are not limited to 


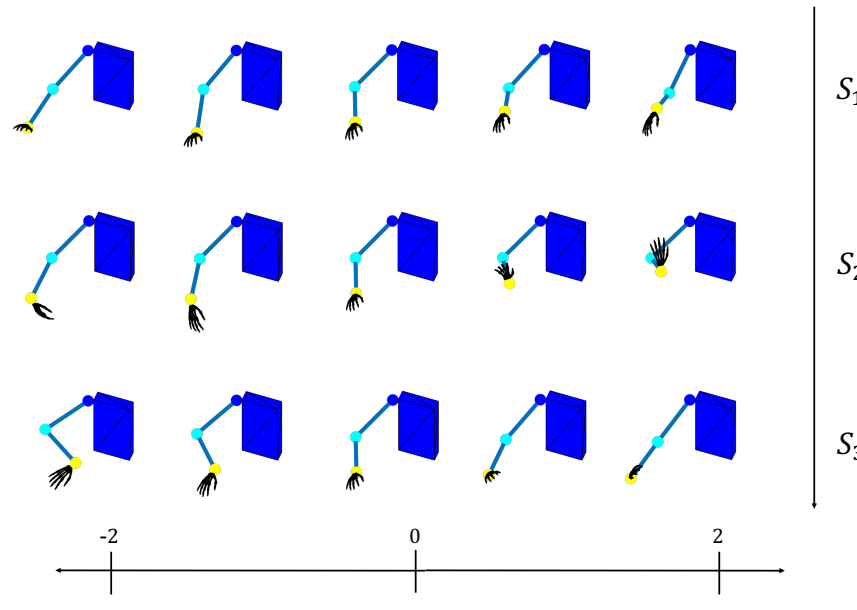

Fig. 6. Different upper limb shapes along the first three synergies. For each row, the central figure shows the mean pose, while the others report the effect of the corresponding synergy modulated by a coefficient $\sigma$ varying between -2 and 2.

the neuroscientific field only, but rather we envision potential applications in robotics as well. The concept of synergies and its intrinsic connection with dimensionality reduction has found fertile application fields in engineering, with special focus on artificial hands and grasping, see the Introduction and e.g. [? 7] for a review on these topics. The time-invariant model described in this work can be used to couple (and underactuate) the DoFs of an artificial upper limb, using mechanical implementation or though software control. This can enable the execution of a large variety of actions while maintaining the actuation complexity low. The same concept, translated in the mechanical domain, could be combined with soft robotics technologies, as it was done e.g. in [42], to instruct the design of simplified robotic manipulators, or prostheses, able to safely and effectively interact with the external environment. At the same time, the PC-based description can be used for simplifying the control of fully-actuated anthropomorphic robotic arms, e.g. for a safe human-robot communication and interaction [62].

\section{CONCLUSIONS}

In this paper, we analyzed human upper limb movements during activities of daily living and described the underlying synergistic behavior. Time-varying synergies have been extracted using a frame-by-frame PCA approach. Results suggest that large part of upper limb kinematic trajectory variability can be approximated, with a good level of accuracy, using a timeinvariant low-dimensional model identified by the first principal components of angular joints. Such description has been proven to be preserved for a large part of the motion execution. Variations of this time-unvarying synergistic description appear in specific portions of the task. We believe that this effect may be related to a fine - likely feedback - control of the limb in the proximity of goal reaching. Further works will follow two main directions: i) include additional tasks or different task set to further strengthen the here reported outcomes, ii) analyze the linkage between these results and the muscular counterpart. Indeed, the relation between kinematic and muscle synergies is still an open point in literature. Recently, some studies attempted to correlate the two domains. In particular, in [47] the authors argued that the relation between muscle and joint-torque patterns is not fixed in general because of the nonlinear relation between the two domains, also considering the intrinsic redundancy of the muscular-skeletal system. Similar findings were discussed in [63] considering hand motion and EMG measurements during reach, grasp and pull tasks. The authors observed that muscle synergies are correlated although shifted in phase whit respect to the kinematic counterpart. The authors also hypothesized that kinematic synergies may have in part origin from a synergistic muscle activation. It is possible to speculate that the description essentially time-invariant of upper limb synergies we reported in this work may be related to principal muscle patterns, while changes observed during the interaction phase may be also introduced by the enrollment of different, time-dependent, muscle synergies in response to environment adaptation.

\section{APPENDIX A \\ LIST OF MOVEMENTS}

Table II lists the 30 tasks included in the proposed protocol. The first column counts the task number, the second links to the corresponding grasp type (here we refer to [49]), the third specify the class of movement (see section III-A) and the fourth column reports a brief description of the task.

\section{REFERENCES}

[1] N. A. Bernstein, "The control and regulation of movements," 1967.

[2] S. Coradeschi, H. Ishiguro, M. Asada, S. C. Shapiro, M. Thielscher, C. Breazeal, M. J. Mataric, and H. Ishida, "Human-inspired robots," IEEE Intelligent Systems, no. 4, pp. 74-85, 2006.

[3] Y.-L. Park, B.-r. Chen, N. O. Pérez-Arancibia, D. Young, L. Stirling, R. J. Wood, E. C. Goldfield, and R. Nagpal, "Design and control of a bio-inspired soft wearable robotic device for ankle-foot rehabilitation," Bioinspiration \&amp; biomimetics, vol. 9, no. 1, p. 016007, 2014.

[4] A. McDaid, K. Kora, S. Xie, J. Lutz, and M. Battley, "Humaninspired robotic exoskeleton (hurex) for lower limb rehabilitation," in Mechatronics and Automation (ICMA), 2013 IEEE International Conference on. IEEE, 2013, pp. 19-24.

[5] S. Kim, C. Laschi, and B. Trimmer, "Soft robotics: a bioinspired evolution in robotics," Trends in biotechnology, vol. 31, no. 5, pp. 287-294, 2013.

[6] S. Casini, V. Tincani, G. Averta, M. Poggiani, C. D. Santina, E. Battaglia, M. G. Catalano, M. Bianchi, G. Grioli, and A. Bicchi, "Design of an under-actuated wrist based on adaptive synergies (accepted)," ICRA, 2017.

[7] M. Santello, M. Bianchi, M. Gabiccini, E. Ricciardi, G. Salvietti, D. Prattichizzo, M. Ernst, A. Moscatelli, H. Jörntell, A. M. Kappers et al., "Hand synergies: integration of robotics and neuroscience for understanding the control of biological and artificial hands," Physics of life reviews, 2016.

[8] I. Jolliffe, Principal component analysis. Springer, 2011.

[9] R. Vinjamuri, M. Sun, C.-C. Chang, H.-N. Lee, R. J. Sclabassi, and Z.-H. Mao, "Temporal postural synergies of the hand in rapid grasping tasks," IEEE Transactions on Information Technology in Biomedicine, vol. 14, no. 4, pp. 986-994, 2010.

[10] G. M. Gasparri, S. Manara, D. Caporale, G. Averta, M. Bonilla, H. Marino, M. Catalano, G. Grioli, M. Bianchi, A. Bicchi et al., "Efficient walking gait generation via principal component representation of optimal trajectories: application to a planar biped robot with elastic joints," IEEE Robotics and Automation Letters, vol. 3, no. 3, pp. 2299-2306, 2018.

[11] V. Catrambone, A. Greco, G. Averta, M. Bianchi, G. Valenza, and E. P Scilingo, "Predicting object-mediated gestures from brain activity: An eeg study on gender differences," IEEE Transactions on Neural Systems and Rehabilitation Engineering, vol. 27, no. 3, pp. 411-418, March 2019.

[12] K. M. Newell and D. E. Vaillancourt, "Dimensional change in motor learning," Human movement science, vol. 20, no. 4-5, pp. 695-715, 2001. 
TABLE II

List OF MOVEMENTS

\begin{tabular}{|c|c|c|c|}
\hline \# & \# Cut & Class & Description \\
\hline 1 & & Int & Ok gesture (lifting hand from the table) \\
\hline 2 & & Int & Thumb down (lifting hand from the table) \\
\hline 3 & & Int & $\begin{array}{l}\text { Exultation (extending the arm up in the air and } \\
\text { keeping it in with closed fist) }\end{array}$ \\
\hline 4 & & Int & $\begin{array}{l}\text { Hitchhiking (extending the arm along the frontal } \\
\text { plane, laterally, parallel to the floor, with extended } \\
\text { elbow, closed fist, extended thumb) }\end{array}$ \\
\hline 5 & & Int & $\begin{array}{l}\text { Block out sun from own face (with open hand, } \\
\text { touching the face with the palm and covering the } \\
\text { eyes) }\end{array}$ \\
\hline 6 & & Int & Greet (with open hand, moving wrist) (3 times) \\
\hline 7 & & Int & Military salute (with lifted elbow) \\
\hline 8 & & Int & $\begin{array}{l}\text { Stop gesture (extending the arm along the sagittal } \\
\text { plane, parallel to the floor, with extended elbow, } \\
\text { open palm) }\end{array}$ \\
\hline 9 & & Int & $\begin{array}{l}\text { Pointing (with index finger) of something straight } \\
\text { ahead (with outstretched arm) }\end{array}$ \\
\hline 10 & & Int & $\begin{array}{l}\text { Silence gesture (bringing the index finger, with } \\
\text { the remainder of the hand closed, on the lips) }\end{array}$ \\
\hline 11 & 2 & $\operatorname{Tr}$ & $\begin{array}{l}\text { Reach and grasp a small suitcase (placed along } \\
\text { own frontal plane) from the handle, lift it and } \\
\text { place it on the floor (close to own chair, along } \\
\text { own sagittal plane) }\end{array}$ \\
\hline 12 & 3 & $\operatorname{Tr}$ & $\begin{array}{l}\text { Reach and grasp a glass, drink for } 3 \text { seconds and } \\
\text { place it in the initial position }\end{array}$ \\
\hline 13 & 4 & $\operatorname{Tr}$ & $\begin{array}{l}\text { Reach and grasp a phone receiver (placed along } \\
\text { own sagittal plane), carry it to own ear for } 3 \\
\text { seconds and place it in the initial position }\end{array}$ \\
\hline 14 & 6 & $\operatorname{Tr}$ & $\begin{array}{l}\text { Reach and grasp a book (placed overhead on a } \\
\text { shelf), put in on the table and open it (from right } \\
\text { side to left side) }\end{array}$ \\
\hline 15 & 8 & $\operatorname{Tr}$ & $\begin{array}{l}\text { Reach and grasp a small cup from the handle ( } 2 \\
\text { fingers }+ \text { thumb), drink for } 3 \text { seconds and place } \\
\text { it in the initial position }\end{array}$ \\
\hline 16 & 11 & $\operatorname{Tr}$ & $\begin{array}{l}\text { Reach and grasp an apple, mimic biting and put } \\
\text { it in the initial position }\end{array}$ \\
\hline 17 & 12,13 & $\operatorname{Tr}$ & $\begin{array}{l}\text { Reach and grasp a hat (placed on the right side of } \\
\text { the table) from its top and place it on own head }\end{array}$ \\
\hline 18 & 12 & $\operatorname{Tr}$ & $\begin{array}{l}\text { Reach and grasp a cup from its top, lift it and put } \\
\text { it on the left side of the table }\end{array}$ \\
\hline 19 & 15 & $\operatorname{Tr}$ & $\begin{array}{l}\text { Receive a tray from someone (straight ahead, with } \\
\text { open hand) and put it in the middle of the table }\end{array}$ \\
\hline 20 & 16 & $\operatorname{Tr}$ & $\begin{array}{l}\text { Reach and grasp a key in a lock (vertical axis), } \\
\text { extract it from the lock and put it on the left side } \\
\text { of the table }\end{array}$ \\
\hline 21 & 1 & T-M & $\begin{array}{l}\text { Reach and grasp a bottle, pour water into a glass } \\
\text { and put the bottle in the initial position }\end{array}$ \\
\hline 22 & $2,3,4$ & T-M & $\begin{array}{l}\text { Reach and grasp a tennis racket (placed along own } \\
\text { frontal plane) and play a forehand (the subject is } \\
\text { still seated) }\end{array}$ \\
\hline 23 & 5 & T-M & $\begin{array}{l}\text { Reach and grasp a toothbrush, brush teeth (hori- } \\
\text { zontal axis, one time left-right) and put it inside } \\
\text { a holder (on the right side of the table) }\end{array}$ \\
\hline 24 & 6 & T-M & $\begin{array}{l}\text { Reach and grasp a laptop, open it (without chang- } \\
\text { ing its position) ( } 4 \text { fingers }+ \text { thumb) }\end{array}$ \\
\hline 25 & $7,8,9$ & T-M & $\begin{array}{l}\text { Reach and grasp a pen (placed on the right side } \\
\text { of the table) and draw a vertical line on the table } \\
\text { (from the top to the bottom) }\end{array}$ \\
\hline 26 & 7 & T-M & $\begin{array}{l}\text { Reach and grasp a pencil (placed along own frontal } \\
\text { plane) ( } 3 \text { fingers }+ \text { thumb) and put it inside a } \\
\text { squared pencil holder (placed on the left side of } \\
\text { the table) }\end{array}$ \\
\hline 27 & 9 & T-M & $\begin{array}{l}\text { Reach and grasp a tea bag in a cup ( } 1 \text { finger }+ \\
\text { thumb), remove it from the cup and place it on } \\
\text { the table on the right side of the table }\end{array}$ \\
\hline 28 & 10 & T-M & $\begin{array}{l}\text { Reach and grasp a doorknob, turn it clockwise } \\
\text { and counterclockwise and open the door }\end{array}$ \\
\hline 29 & 13 & T-M & $\begin{array}{l}\text { Reach and grasp a tennis ball (with fingertips) and } \\
\text { place it in a basket on the floor (right) }\end{array}$ \\
\hline 30 & 14 & T-M & $\begin{array}{l}\text { Reach and grasp a cap ( } 2 \text { fingers }+ \text { thumb) of a } \\
\text { bottle (held by left hand), unscrew it and place it } \\
\text { overhead on a shelf }\end{array}$ \\
\hline
\end{tabular}

[13] M. L. Latash, J. P. Scholz, and G. Schöner, "Toward a new theory of motor synergies," Motor control, vol. 11, no. 3, pp. 276-308, 2007.

[14] J. P. Scholz and G. Schöner, "The uncontrolled manifold concept: identifying control variables for a functional task," Experimental brain research, vol. 126, no. 3, pp. 289-306, 1999.

[15] B. Tuller and M. Turvey, "The bernstein perspective: 1l. the concept of muscle linkage or coordinative," Human motor behavior: An introduction, p. $253,1982$.

[16] G. E. Loeb, “Optimal isn't good enough,” Biological cybernetics, vol. 106, no. 11-12, pp. 757-765, 2012.

[17] T. Flash and N. Hogan, "The coordination of arm movements: an experimentally confirmed mathematical model," Journal of neuroscience, vol. 5, no. 7, pp. 1688-1703, 1985.

[18] M. Santello, M. Flanders, and J. F. Soechting, "Postural hand synergies for tool use," The Journal of Neuroscience, vol. 18, no. 23, pp. $10105-$ $10115,1998$.

[19] — , "Patterns of hand motion during grasping and the influence of sensory guidance," Journal of Neuroscience, vol. 22, no. 4, pp. 14261435, 2002.

[20] A. d'Avella, P. Saltiel, and E. Bizzi, "Combinations of muscle synergies in the construction of a natural motor behavior," Nature neuroscience, vol. 6, no. 3, p. 300, 2003.

[21] P. Saltiel, K. Wyler-Duda, A. D'Avella, M. C. Tresch, and E. Bizzi, "Muscle synergies encoded within the spinal cord: evidence from focal intraspinal nmda iontophoresis in the frog," Journal of neurophysiology, vol. 85, no. 2, pp. 605-619, 2001.

[22] L. H. Ting and J. M. Macpherson, "A limited set of muscle synergies for force control during a postural task," Journal of neurophysiology, 2005.

[23] M. C. Tresch, P. Saltiel, and E. Bizzi, "The construction of movement by the spinal cord," Nature neuroscience, vol. 2, no. 2, p. 162, 1999.

[24] V. Krishnamoorthy, S. Goodman, V. Zatsiorsky, and M. L. Latash, "Muscle synergies during shifts of the center of pressure by standing persons: identification of muscle modes," Biological cybernetics, vol. 89, no. 2, pp. 152-161, 2003.

[25] M. C. Tresch, V. C. Cheung, and A. d'Avella, "Matrix factorization algorithms for the identification of muscle synergies: evaluation on simulated and experimental data sets," Journal of neurophysiology, 2006.

[26] A. d Avella and M. C. Tresch, "Modularity in the motor system: decomposition of muscle patterns as combinations of time-varying synergies," Advances in neural information processing systems, vol. 1, pp. 141-148, 2002.

[27] E. J. Weiss and M. Flanders, "Muscular and postural synergies of the human hand," Journal of neurophysiology, 2004.

[28] C. Castellini and P. van der Smagt, "Evidence of muscle synergies during human grasping," Biological cybernetics, vol. 107, no. 2, pp. 233-245, 2013.

[29] F. J. Valero-Cuevas, "Predictive modulation of muscle coordination pattern magnitude scales fingertip force magnitude over the voluntary range," Journal of Neurophysiology, vol. 83, no. 3, pp. 1469-1479, 2000.

[30] M. Maier and M. Hepp-Reymond, "Emg activation patterns during force production in precision grip. ii. muscular synergies in the spatial and temporal domain." Experimental brain research, vol. 103, no. 1, pp. 123-136, 1995.

[31] B. Poston, A. Danna-Dos Santos, M. Jesunathadas, T. M. Hamm, and M. Santello, "Force-independent distribution of correlated neural inputs to hand muscles during three-digit grasping," American Journal of Physiology-Heart and Circulatory Physiology, 2010.

[32] A. d'Avella, A. Portone, L. Fernandez, and F. Lacquaniti, "Control of fast-reaching movements by muscle synergy combinations," Journal of Neuroscience, vol. 26, no. 30, pp. 7791-7810, 2006.

[33] Y. P. Ivanenko, R. E. Poppele, and F. Lacquaniti, "Five basic muscle activation patterns account for muscle activity during human locomotion," The Journal of physiology, vol. 556, no. 1, pp. 267-282, 2004.

[34] J. Roh, W. Z. Rymer, E. J. Perreault, S. B. Yoo, and R. F. Beer, "Alterations in upper limb muscle synergy structure in chronic stroke survivors," American Journal of Physiology-Heart and Circulatory Physiology, 2012.

[35] A. Schwarz, G. Averta, J. M. Veerbeek, A. Luft, J. P. Held, G. Valenza, A. Bicchi, and M. Bianchi, "A functional analysis-based approach to quantify upper limb impairment level in chronic stroke patients: A pilot study," in Engineering in Medicine and Biology Society, 2019. EMBC'19. 41th Annual International Conference of the IEEE. IEEE, 2019.

[36] L. Dipietro, H. I. Krebs, S. E. Fasoli, B. T. Volpe, J. Stein, C. T. Bever, and N. Hogan, "Changing motor synergies in chronic stroke," Journal of neurophysiology, 2007. 
[37] A. d'Avella, M. Giese, Y. P. Ivanenko, T. Schack, and T. Flash, "Modularity in motor control: from muscle synergies to cognitive action representation," Frontiers in computational neuroscience, vol. 9, p. 126, 2015.

[38] C. Della Santina, M. Bianchi, G. Averta, S. Ciotti, V. Arapi, S. Fani, E. Battaglia, M. G. Catalano, M. Santello, and A. Bicchi, "Postural hand synergies during environmental constraint exploitation," Frontiers in neurorobotics, vol. 11, p. 41, 2017.

[39] C. R. Mason, J. E. Gomez, and T. J. Ebner, "Hand synergies during reachto-grasp," Journal of Neurophysiology, vol. 86, no. 6, pp. 2896-2910, 2001.

[40] P. H. Thakur, A. J. Bastian, and S. S. Hsiao, "Multidigit movement synergies of the human hand in an unconstrained haptic exploration task," The Journal of neuroscience, vol. 28, no. 6, pp. 1271-1281, 2008.

[41] G. Averta, F. Angelini, M. Bonilla, M. Bianchi, and A. Bicchi, "Incrementality and hierarchies in the enrollment of multiple synergies for grasp planning," IEEE Robotics and Automation Letters, vol. 3, no. 3, pp. 2686-2693, 2018.

[42] M. G. Catalano, G. Grioli, E. Farnioli, A. Serio, C. Piazza, and A. Bicchi, "Adaptive synergies for the design and control of the pisa/iit softhand," The International Journal of Robotics Research, vol. 33, no. 5, pp. 768-782, 2014.

[43] O. Heidari, J. O. Roylance, A. Perez-Gracia, and E. Kendall, "Quantification of upper-body synergies: A case comparison for stroke and non-stroke victims," in ASME 2016 International Design Engineering Technical Conferences and Computers and Information in Engineering Conference. American Society of Mechanical Engineers, 2016, pp. V05AT07A032-V05AT07A032.

[44] D. S. Reisman and J. P. Scholz, "Aspects of joint coordination are preserved during pointing in persons with post-stroke hemiparesis," Brain, vol. 126, no. 11, pp. 2510-2527, 2003.

[45] E. E. Butler, A. L. Ladd, S. A. Louie, L. E. LaMont, W. Wong, and J. Rose, "Three-dimensional kinematics of the upper limb during a reach and grasp cycle for children," Gait \&amp; posture, vol. 32, no. 1, pp. 72-77, 2010.

[46] A. d'Avella, A. Portone, L. Fernandez, and F. Lacquaniti, "Control of fast-reaching movements by muscle synergy combinations," The Journal of neuroscience, vol. 26, no. 30, pp. 7791-7810, 2006.

[47] M. Russo, M. D'Andola, A. Portone, F. Lacquaniti, and A. d'Avella, "Dimensionality of joint torques and muscle patterns for reaching," Frontiers in computational neuroscience, vol. 8, p. 24, 2014.

[48] E. Todorov and Z. Ghahramani, "Analysis of the synergies underlying complex hand manipulation," in Engineering in Medicine and Biology Society, 2004. IEMBS'04. 26th Annual International Conference of the IEEE, vol. 2. IEEE, 2004, pp. 4637-4640.

[49] M. R. Cutkosky, "On grasp choice, grasp models, and the design of hands for manufacturing tasks," IEEE Transactions on robotics and automation, vol. 5, no. 3, pp. 269-279, 1989.

[50] T. Feix, J. Romero, H.-B. Schmiedmayer, A. M. Dollar, and D. Kragic, "The grasp taxonomy of human grasp types," IEEE Transactions on Human-Machine Systems, vol. 46, no. 1, pp. 66-77, 2016.

[51] J. Lenarcic and A. Umek, "Simple model of human arm reachable workspace," IEEE transactions on systems, man, and cybernetics, vol. 24, no. 8, pp. 1239-1246, 1994.

[52] K. Abdel-Malek, J. Yang, R. Brand, and E. Tanbour, "Towards understanding the workspace of human limbs," Ergonomics, vol. 47, no. 13, pp. 1386-1405, 2004.

[53] J. C. Perry, J. Rosen, and S. Burns, "Upper-limb powered exoskeleton design," IEEE/ASME transactions on mechatronics, vol. 12, no. 4, p. 408, 2007.

[54] R. Cubelli, C. Marchetti, G. Boscolo, and S. Della Sala, "Cognition in action: Testing a model of limb apraxia," Brain and cognition, vol. 44 no. 2, pp. 144-165, 2000.

[55] M. Benati, S. Gaglio, P. Morasso, V. Tagliasco, and R. Zaccaria, "Anthropomorphic robotics," Biological Cybernetics, vol. 38, no. 3, pp. $125-140,1980$.

[56] K. R. Holzbaur, W. M. Murray, and S. L. Delp, "A model of the upper extremity for simulating musculoskeletal surgery and analyzing neuromuscular control," Annals of biomedical engineering, vol. 33, no. 6 , pp. 829-840, 2005.

[57] G. Averta, C. Della Santina, E. Battaglia, F. Felici, M. Bianchi, and A. Bicchi, "Unvealing the principal modes of human upper limb movements through functional analysis," Frontiers in Robotics and AI, vol. 4, p. 37, 2017.

[58] D. J. Berndt and J. Clifford, "Using dynamic time warping to find patterns in time series." in $K D D$ workshop, vol. 10, no. 16. Seattle, WA, 1994 pp. 359-370.
[59] S. Salvador and P. Chan, "Toward accurate dynamic time warping in linear time and space," Intelligent Data Analysis, vol. 11, no. 5, pp. 561-580, 2007.

[60] A. V. Knyazev and M. E. Argentati, "Principal angles between subspaces in an a-based scalar product: algorithms and perturbation estimates," SIAM Journal on Scientific Computing, vol. 23, no. 6, pp. 2008-2040, 2002.

[61] M. Santello and C. E. Lang, "Are movement disorders and sensorimotor injuries pathologic synergies? when normal multi-joint movement synergies become pathologic," Frontiers in human neuroscience, vol. 8, p. 1050, 2015.

[62] B. R. Duffy, "Anthropomorphism and the social robot," Robotics and autonomous systems, vol. 42, no. 3, pp. 177-190, 2003.

[63] M. Tagliabue, A. L. Ciancio, T. Brochier, S. Eskiizmirliler, and M. A. Maier, "Differences between kinematic synergies and muscle synergies during two-digit grasping," Frontiers in human neuroscience, vol. 9, p. 165,2015 